\title{
Dimension intertrial and cueing effects in localization: support for pre-attentively weighted one-route models of saliency
}

\author{
Michael Zehetleitner • Joseph Krummenacher • \\ Thomas Geyer • Michael Hegenloh • Hermann J. Müller
}

Published online: 6 November 2010

(C) Psychonomic Society, Inc. 2010

\begin{abstract}
There are several alternative accounts of dimensional intertrial and cueing effects in singleton feature search tasks. Some accounts assume that these effects arise at post-selective processing stages; dual-route accounts assume them to be perceptual in nature, but coming into play only in non-spatial tasks (e.g., detection but not localization). By contrast, the Dimension Weighting Account (DWA) assumes dimensional effects to arise at pre-attentive processing stages of spatial as well as non-spatial tasks. The data available are ambiguous, permitting no clear-cut choice among these accounts. Therefore, the present study examined for early effects of dimensional weighting in a spatial task, the presence of which is only predicted by the DWA and not by post-selective or dual-route accounts. Salience is known to saturate for high feature contrast and long presentation times. Consequently, with lower bottom-up salience that still permits efficient search, dimensional weights would produce a greater modulation - if present at all. Thus, we examined localization accuracy under brief-presentation conditions in Experiment 1, and localization speed under conditions of low versus high feature contrast in Experiment 2. Both experiments revealed
\end{abstract}

M. Zehetleitner · T. Geyer • M. Hegenloh • H. J. Müller

Ludwig-Maximilians-Universität München,

München, Germany

J. Krummenacher • H. J. Müller

Université de Fribourg,

Fribourg, Switzerland

H. J. Müller

Birkbeck College University of London,

London, UK

M. Zehetleitner $(\bowtie)$

LMU Department Psychologie,

Leopoldstr. 1380802 München, Germany

e-mail: mzehetleitner@psy.lmu.de significant dimension intertrial and cueing effects. This strongly argues against dual-route accounts and strengthens evidence for a pre-attentive origin of these effects.

Keywords Singleton feature targets · Visual-search · Topdown - Attentional guidance - Stimulus-driven · Pop-out . Selection $\cdot$ Dimension $\cdot$ Features $\cdot$ Localization

\section{Introduction}

The Dimension Weighting Account (DWA; Found \& Müller, 1996; Müller, Heller, \& Ziegler, 1995) provides an extension to saliency summation models (e.g., Bruce \& Tsotsos, 2009; Gao, Mahadevan, \& Vasconcelos, 2008; Itti \& Koch, 2001; Koch \& Ullman, 1985; Wolfe, 1994) devised to explain a number of phenomena in visual search. Saliency summation models assume that the allocation of focal attention to the search target is guided by an overallsaliency map of the visual array, with the saliency map units summing, in a location-specific manner, feature contrast signals computed in separate feature dimensions (such as color, orientation, etc.). Importantly, feature contrast and (overall-) saliency activations signal only the presence of feature differences at certain locations in the search array, without specifying the underlying features giving rise to them. Currently, the literature suggests two competitor models to the DWA: the Dimension Action Model (Cohen \& Magen, 1999; Cohen \& Shoup, 1997, 2000) and augmented Feature Integration Theory (FIT: Chan, \& Hayward, 2009; Treisman \& Gelade, 1980; Treisman, \& Gormican, 1988). The three models are dissociable in terms of their predictions regarding the topdown and bottom-up modulations of performance in search tasks that require coarse localization (left vs right) of feature singletons in the visual array. In feature singleton 
search, the target is defined by a simple feature difference relative to the non-targets, such as a red vertical bar amongst green vertical bars (color-defined target), and, typically, the target definition is randomly variable across trials (e.g., a color-defined target may be followed by an orientationdefined target, such as a green right-tilted bar amongst green vertical bars). Differential predictions made by the three alternative models with regard to localization performance in singleton feature search are examined in the present study.

There are two findings in the search literature that are not readily explained by simple summation of feature contrast signals (Itti \& Koch, 2001; Wolfe, 1994). First, in tasks requiring detection of feature singletons, search performance is not ahistoric. Rather, search reaction times (RTs) are slower and accuracy lower when the targetdefining dimension is changed, rather than repeated, across consecutive trials (Found \& Müller, 1996; dimension intertrial effects; e.g., Müller, Krummenacher, \& Heller, 2004). Second, in addition to such (largely) passive intertrial effects, search performance can also be actively modulated by the observer's top-down intentions. For example, Müller, Reimann and Krummenacher (2003) examined how trial-by-trial semantic pre-cueing of the likely defining dimension of an upcoming target would influence search performance in a singleton detection task. The cue words could be either valid (e.g., cue 'color' followed by a color-defined singleton) or invalid (e.g., cue 'color' followed by an orientation -defined singleton), or neutral (cue word 'neutral' which was equally likely to be followed by a color- or an orientation-defined target). RTs were found to be faster with valid as compared to neutral (and invalid) pre-cues, and slower with invalid as compared to neutral (and valid) cues (dimensional cueing benefits and costs). That is, search performance was modulated by observers actively setting themselves for the likely target dimension.

\section{The dimension weighting account}

The DWA accounts for passive, bottom-up and active, topdown effects on search performance by dimension changes/ repetitions across trials and, respectively, trial-by-trial precues by assuming a pre-selective modulation of feature contrast signals via adjustable dimensional weights: the relative weights assigned to possible target dimensions modulate the processing of feature contrast signals prior to their integration by the (overall-) saliency units, that is, before focal-attentional selection takes place (see Fig. 1). The crucial notion is that higher weights for one dimension enhance the build-up of activation on the saliency map driven by feature contrast in the weighted dimension, which in turn increases discriminability of targets from distractors on that map (both in terms of speed and signal-to-noise ratio), thereby improving search performance. To explain the two effects outlined above, the DW model further assumes that the dimensional weights are modulable in two distinct ways: bottom-up (passively) by the nature of the target event on the previous trial(s), and top-down (actively) by observers' intentions. After encountering a target defined in a particular dimension (e.g., color), the corresponding dimensional weight (i.e., that for color) is automatically increased, whereas all other dimensional weights (e.g., for orientation, motion, etc.) are decreased; this gives rise to facilitated search performance after repetitions, compared to changes, of the target-defining dimension. If an observer actively prepares for a specific dimension in response to a pre-cue, the same weighting process is assumed to be operational, that is, the weight is increased for the pre-cued dimension, while all other dimensional weights are decreased simultaneously; this accounts for dimensional cueing benefits and costs. Importantly, similar to the GS model, the DW account is a one-route model that assumes that the (overall-) saliency map is engaged in all tasks involving processing of feature contrast signals, that is, simple detection tasks as well as compound-search and localization tasks (see below).

\section{Alternative accounts}

However, the DWA has not gone unchallenged. Alternative models argue either against the pre-selective locus of the dimension-dependent performance modulations, or against the one-route assumption that spatial (e.g., compound and localization) as well as non-spatial (e.g., detection) tasks are similarly processed via the overall-saliency map. The challenges are based on several null findings: (absent) dimension repetition/change effects or (absent) benefits and costs from dimensional cues - the two key effects that are taken by the DWA to reflect bottom-up and, respectively, top-down modulations of pre-selective dimensional weight settings (Table 1).

Data basis To start with, Theeuwes, Reimann, Mortier, \& Karen (2006) investigated the effect of semantic dimension cues on search performance in simple detection and compound-search tasks. In the former, observers simply respond target-present upon detecting a singleton item, for example, a color-defined target. In the latter, observers also need to detect the singleton, but the required response is based on some feature other than that singling out the target from amongst the distractors, for example, respond to the orientation of a small line inside the odd-colored object (Bravo \& Nakayama, 1992; Duncan, 1985). Logically, as the distractors also contain small lines inside that are either response-compatible or -incompatible with the target line, this task requires localization and focal-attentional processing of the target in order to discern the response-critical 
Fig. 1 The dimension weighting account assumes that visual input (light gray representing green, and black representing red bars) is analyzed in terms of its features in different feature maps, which are summed into dimension maps. The activity of dimension maps is modulated by dimensional weights for color, orienation, and motion $\left(w_{c}, w_{o}\right.$, and $\left.w_{m}\right)$ before being integrated into the master saliency map

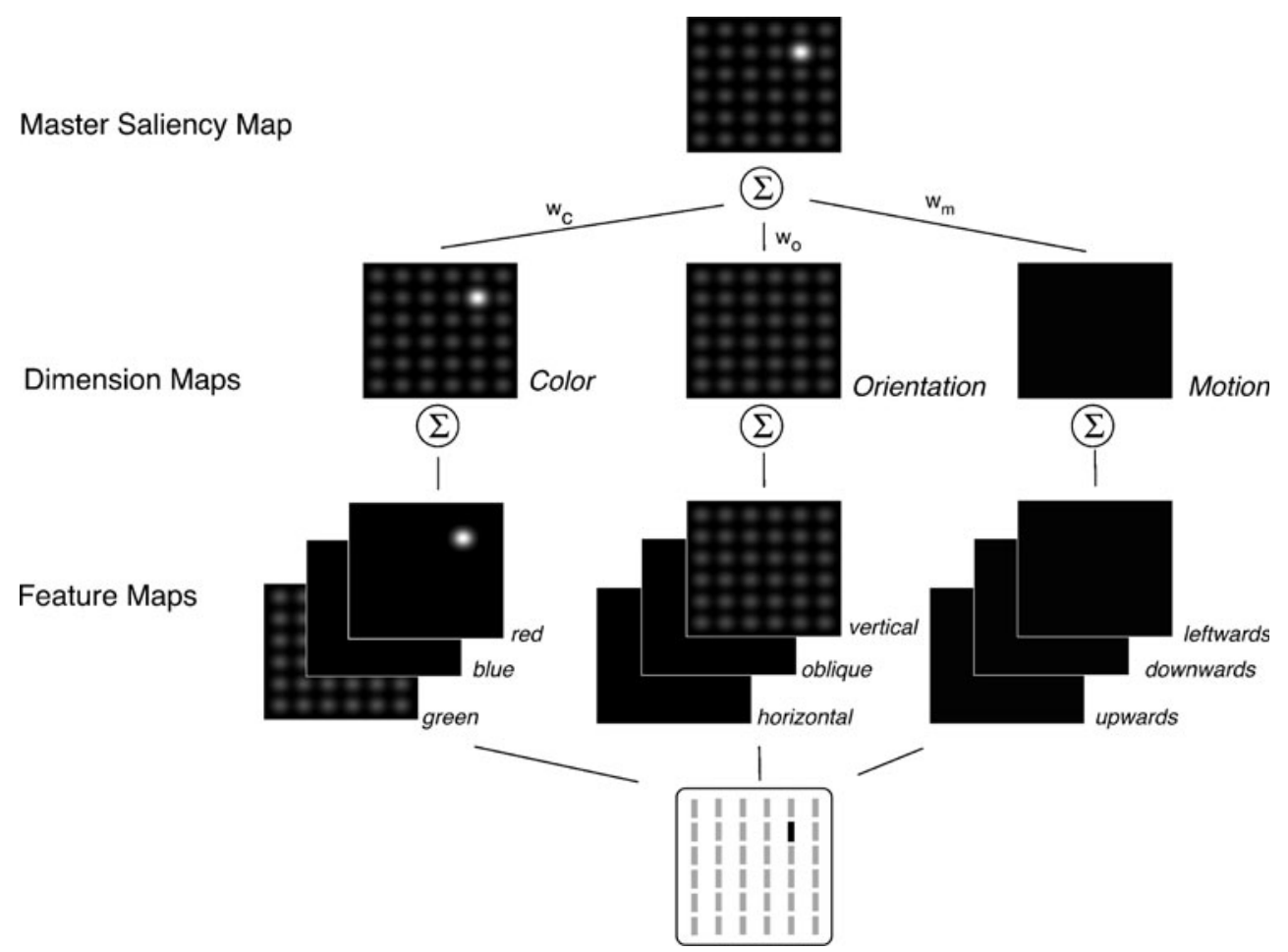

attribute. While Theeuwes et al. (2006) could replicate Müller et al.'s (2003) finding of facilitated performance for valid- compared to invalid-dimension pre-cue trials in a detection task, they failed to find statistically significant benefits and costs from the same semantic pre-cues in a compound task.

Second, Chan and Hayward (2009) investigated dimension repetition/change effects in spatial (that is, compound and localization) as well as non-spatial (that is, detection) tasks; the latter may be regarded as non-spatial because target

Table 1 Summary of studies employing a spatial task that did find or failed to find significant dimension intertrial (DI) and dimensional cueing (DC) effects in compound tasks

\begin{tabular}{llll}
\hline Study & \multicolumn{2}{l}{ Type of effect } & \multirow{2}{*}{ Task } \\
\cline { 2 - 3 } & DI & DC & \\
\hline Theeuwes et al. (2006) & Yes & No & Compound \\
Müller \& Krummenacher (2006) & Yes & Yes & Compound \\
Theeuwes \& van der Burg (2007) & n.a. ${ }^{a}$ & No & Compound \\
Töllner et al. (2008) & Yes & n.a. ${ }^{\text {b }}$ & Compound \\
Chan \& Hayward (2009) & No & n.a. ${ }^{\text {b }}$ & Compound \\
Chan \& Hayward (2009) & No & n.a. ${ }^{b}$ & Localization \\
Mortier et al. (2010) & n.a. ${ }^{\text {a }}$ & No & Localization \\
Töllner et al. (2010) & Yes & Yes & Compound \\
\hline
\end{tabular}

${ }^{\mathrm{a}}$ Not reported

${ }^{\mathrm{b}}$ No word cues in paradigm localization is not strictly necessary to make a target-present response. Chan and Hayward were able to replicate dimension repetition/change effects for the detection task (Found \& Müller, 1996), but they failed to find statistically significant dimensional intertrial effects in localization and compound tasks. Additionally, Mortier, Zoest, Meeter, and Theeuwes (2010) found dimensional cues to be effective only in detection, but not in localization, tasks, whether responses were manual or ocular. Finally, (Theeuwes \& van der Burg, 2007) investigated symbolic spatial and symbolic dimensional cueing effects in a compound task under brief viewing conditions, examining the signal detection measure A'. They reported only spatial, but not dimensional cues to improve accuracy in discriminating the orientation of a line segment located inside a color or form singleton; concerning dimension cuing, there was a small, though non-reliable advantage for $100 \%$ valid versus 50\% neutral cues in Experiment $3(.88$ vs .86), and for $80 \%$ valid versus $20 \%$ invalid cues in Experiment $4(.81$ vs .86; $F(1,13)=1.55, p=.235)$.

These null-effects are unexpected from the DWA, but are in accordance with post-selective weighting accounts and dual-route search architectures: Detection tasks on the one hand and compound and localization tasks on the other differ in two respects: the requirement of spatial information and the stimulus-response mapping(s). On this basis (which is elaborated below), these null findings were taken to support models that argue in favor of a post-selective, rather than a pre-selective, locus of dimension weighting effects and/or for a dual-route account that assumes 
different processing paths for non-spatial detection and spatial localization and compound tasks.

Post-selective accounts A representative post-selective model has been proposed in the Dimension Action Model (DAM) of Cohen and colleagues (Cohen \& Feintuch, 2002; Cohen \& Magen, 1999; Cohen \& Shoup, 1997; Mortier, Theeuwes, \& Starreveld, 2005). The DAM assumes that there are dimension-specific feature analyzer units as well as multiple response selection units, one per visual dimension. While the dimensional response selection units compute responses in parallel, the response decision of only one such unit can be transferred to an executive (workingmemory) stage which mediates overt reactions. According to the DAM, selecting a response module, for transfer of its activity to the executive stage, requires focal attention. Cohen and Magen (1999) proposed that the dimensional response selection units are weighted depending on the relevant dimension on the previous trial. Accordingly, dimension-specific (bottom-up as well as top-down) effects should occur only when the feature analyzer and the response selection units correlate. This is the case in detection tasks, as the same response button has to be pressed to all targets, whatever their defining dimension, target presence and response selection unit always coincide. By contrast, if the target- and the response-defining features are dissociated, weighting of (target-) dimension-specific response modules should be rendered ineffective. This is the case, for example, in compound-search or localization tasks, where the detection-critical feature of the target is different from the response-critical feature.

Dual-route accounts The null-findings of dimensionspecific intertrial effects and dimensional cueing effects in spatial tasks have also been taken as supportive evidence for another alternative to the pre-attentive, oneroute DWA, as advocated recently by Chan and Hayward (2009) in a 'revival' of FIT (see also Mortier et al., 2010). They assume that detection tasks are not processed via an overall-saliency map but rather via 'dimensional modules' that signal only the presence of dimension-specific feature contrast, but do not convey spatial information (a concept originally proposed by Treisman; e.g., Evans \& Treisman, 2005; Treisman \& Gelade, 1980; Treisman \& Gormican, 1988; see also Müller \& Krummenacher, 2006). By contrast, spatial (i.e., compound-search and localization) tasks are processed via the saliency map. According to this dual-route account, only the dimensional modules are subject to dimensional weighting, but not the computation of the saliency map (Fig. 2). Consequently, dimensional weighting only affects non-spatial detection, but not spatial localization or compound-task performance (a dissociation reported by Chan \& Hayward, 2009, and
Mortier et al., 2010; see also Theeuwes \& van der Burg, 2007).

Purpose of the present study

Although all three models make clear-cut predictions, the available evidence is equivocal. Contrary to the predictions of the DWA, there have been reports of null findings concerning dimension-specific intertrial effects (e.g., Becker, 2008a; Chan \& Hayward, 2009; Kumada, 2001) as well as dimensional cueing effects (Theeuwes et al., 2006) in compound-search tasks. On the other hand, there are also reports of dimensional intertrial effects (Müller \& Krummenacher, 2006; Theeuwes et al., 2006; Töllner, Gramann, Müller, Kiss, \& Eimer, 2008 and cueing effects (Müller \& Krummenacher, 2006; Töllner, Zehetleitner, Gramann, \& Müller, 2010) in studies that have used the very same type of task (see Table 1). Given this, the present study was designed to re-examine both the question of the locus (pre-selective or post-selective?) and that of the processing architecture (oneroute or dual-route?) with respect to dimensional (bottom-up) intertrial sequence and (top-down) cueing effects. To decide among the alternative accounts, coarse localization (left vs right half of the display) of a feature singleton target was used. The (pre-selective, one-route) DWA predicts both dimension-specific intertrial and dimensional cueing effects in such a task. By contrast, post-selective as well as dualroute models would predict neither intertrial nor cueing effects. In localization tasks, the target- and responsedefining features are independent (Bravo \& Nakayama, 1992; Duncan, 1985), rendering post-selective dimension weighting mechanisms ineffective. Therefore, post-selective weighting accounts such as DAM would predict no dimension repetition/change or cueing effects in localization tasks. Furthermore, as spatial information is necessary to solve the task (which is provided only by the overall-saliency map route, but not by the dimensional-modules route assumed in 'revived' FIT; see Fig. 2), dual-route accounts would assume that performance is mediated via the saliency map. Consequently, because the computation of this map is 'unweighted', dual-route accounts would neither predict dimensional intertrial effects nor cueing effects to be observed in this task.

As stated above, support for the DAM and dual-route accounts is mostly based on dimensional effects being absent in spatial tasks. However, these null findings can have two reasons: either dimensional effects cannot, in principle, occur in such tasks (as is assumed by the DAM and dual-route accounts), or the effects are potentially observable but did not become manifest for some (as yet unspecified) reasons. Proceeding from the latter alternative, the present study aimed at realizing conditions that would 
Fig. 2 The dual-route model of Chan \& Hayward (2009) also assumes a saliency map, but in contrast to the DW model, not dimension maps are weighted, but dimensional modules, which represent only presence of feature contrast in as specific dimension over the whole visual field, that is without any spatial information

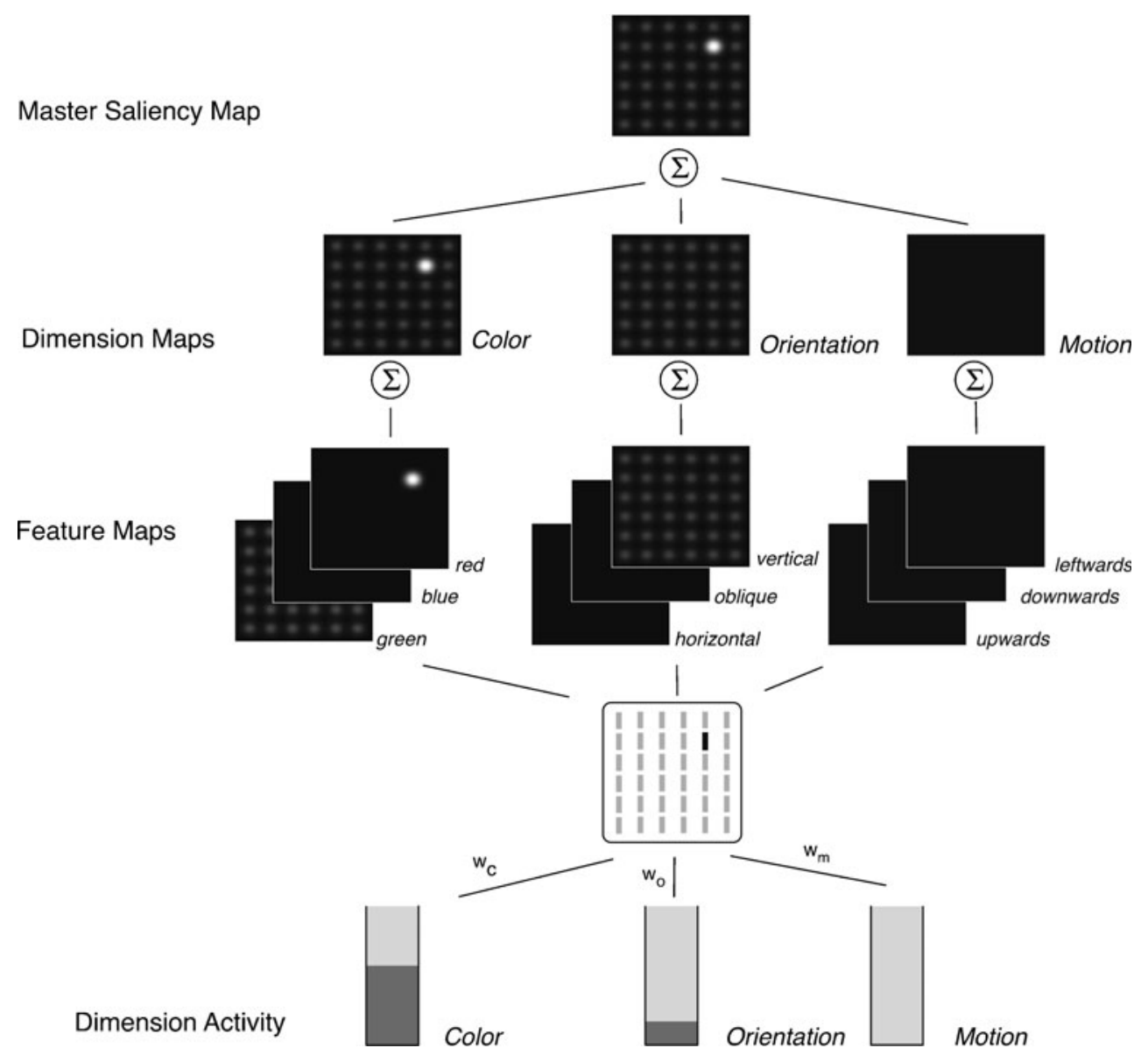

be more conducive for dimensional effects to be observed. If dimensional intertrial effects still fail to manifest under these conditions, this would strengthen the case that such effects are really not there. However, if dimensional effects are demonstrable under these conditions, this would falsify all models predicting that such effects cannot occur due to nature of the processing architecture. The latter outcome, though, would leave it open exactly what the difference is between non-spatial detection and spatial tasks which is responsible for the failure of (some of) the studies reviewed above to find dimensional effects.

In order to increase the likelihood of observing dimensional effects in a localization task, the present study made use of the asymptotic behavior of salience. With strong feature contrast and long presentation times, effective salience would be approaching an asymptotic level (saturation asymptote; Gao et al., 2008; Nothdurft, 1993, 2000) where any additional modulations by dimensional weighting would yield only a diminishing effect. Therefore, to increase the chance of reliably measuring dimensional weighting effects in the present study, conditions of reduced salience were realized by presenting stimulus displays only briefly in Experiment 1 and by decreasing feature contrast (while still ensuring efficient search) in Experiment 2.
Justification for the effectiveness of this approach comes from Zehetleitner, Krummenacher, and Müller (2009) who have already shown that manipulation of feature contrast modulates the size of a different salience-based effect - namely, enhanced search speed for singleton targets that differ from distractors redundantly in two dimensions, rather than in just one dimension (so-called redundancy gains) - in a detection task; the redundancy gains were larger for targets of low compared to high feature contrast (with even the former targets being detected efficiently). Since the non-redundant targets in this study were, variably across trials, defined in either one or another dimension (luminance or, respectively, orientation), it became possible to examine, besides redundancy gains (see Zehetleitner, Proulx, \& Müller, 2009), the size of the dimensional intertrial effects in a re-analysis of the data: this analysis revealed the intertrial effects to also be larger for low- compared to high-feature-contrast targets: $28 \mathrm{~ms}$ versus $10 \mathrm{~ms}$ (significant feature contrast $\mathrm{x}$ dimension transition interaction: $F(1,14)=6.2, p<.025)$. Thus, given that the level of feature contrast modulates dimensional intertrial effects in detection tasks, it would be expected to do the same in a localization task, provided that localization decisions are mediated by the same (single) route as detection decisions. 
To further investigate the pre-selective, perceptual locus of dimensional intertrial and cueing effects (as opposed to a post-selective, response-related locus), the dependent measure in the present study was performance accuracy (under limited stimulus exposure conditions), as well as RT (under unlimited viewing conditions). RT measures are influenced by both pre-selective and post-selective processing stages. However, only perceptual processing stages can increase the quality of the sensory input and thus lead to modulations of accuracy measures under time-limited viewing conditions (Prinzmetal, McCool, \& Park, 2005; Santee \& Egeth, 1982). Accordingly, experimental manipulations that lead to performance modulations in both response speed under unlimited viewing conditions and accuracy under limited viewing conditions can be considered as pre-selective, whereas manipulations that lead only to RT modulations under unlimited viewing conditions have to be considered post-selective. This logic has recently been applied in a number of studies designed to examine for contributions of early perceptual versus later responserelated processing stages to performance in visual search (e.g., Huang \& Pashler, 2005; Sigurdardottir, Kristjansson, \& Driver, 2008).

Based on this logic, in the present Experiment 1, search displays were presented only briefly and followed by a mask. If dimensional effects arise at a perceptual processing stage, (1) repetition of the target-defining dimension should lead to higher (localization) accuracy compared to dimensional change, and (2) valid dimension pre-cues should increase accuracy compared to neutral and/or invalid cues. Experiment 2 measured RTs without limitations on viewing time, in order to examine whether dimensional effects are increased for lower levels of salience.

\section{Experiment 1}

In Experiment 1, the task was to report the location, left versus right visual hemi-field, of a feature singleton that could be defined by either orientation (left- or right-tilted bar amongst vertical bars) or luminance (light gray bar amongst dark gray bars). The display was presented briefly and followed by a mask. The target dimension could change randomly from trial to trial. There were three different types of symbolic cue: neutral, valid, and invalid, which probabilistically indicated the dimension of the upcoming target.

\section{Method}

Participants A total of 24 observers participated in Experiment 1 (13 male; three left-handed; normal or corrected-tonormal vision; median age 24 years), in return for a course credit.
Apparatus The stimuli were presented on a Sony Multiscan E250 17 inch (ca. $43.7 \mathrm{~cm}$ ) monitor driven by a personal computer (PC) with Windows XP operating system, placed in a sound-isolated room with black interior and a dim background light in order to prevent reflections on the monitor. The experimental software was purpose-written in $\mathrm{C}++$. Viewing distance was held constant $($ at $60 \mathrm{~cm}$ ) with aid of a chin rest. Observers reported target location by pressing the right or left button of a mouse with the index or middle finger of their right hand. RTs and accuracy were recorded online by the PC. After each block, observers were informed about their performance (mean RT and error rate) in the previous block.

Stimuli and timing The display consisted of a $6 \times 6$ array (subtending $15.3 \times 15.3^{\circ}$ of visual angle) of filled upright rectangles (bars) on a black background $\left(0.6 \mathrm{~cd} / \mathrm{m}^{2}\right)$. The bars were either dark $(11.6 \mathrm{~cd} / \mathrm{m} 2)$ or light gray; the luminance of the light gray bars was adjusted individually for each observer (see below) and ranged from 60 to $90 \mathrm{~cd} / \mathrm{m}^{2}$. The bars subtended $1.7^{\circ}$ of visual angle in height and $0.35^{\circ}$ in width. They were arranged within invisible array cells sized $2.55 \times 2.55^{\circ}$, with a random jitter of about $0.2^{\circ}$ around the (invisible) center of a cell. Distractors were all medium gray $\left(60 \mathrm{~cd} / \mathrm{m}^{2}\right)$ vertical bars. The target was defined in either the luminance (light gray) or the orientation dimension $\left(45^{\circ}\right.$ tilted to the left or right of vertical). Targets were always placed in such a way that they were surrounded by distractors (i.e., they appeared in the inner $4 \times 4$ cells of the matrix); observers were not informed of this restriction. A target was present in $100 \%$ of the trials, and was placed randomly in the left or the right half of the array. There were three types of cue: neutral, valid, and invalid. Neutral cues were presented in separate blocks.

Trials started with the German words for 'neutral', 'orientation', or 'luminance', which were displayed for $800 \mathrm{~ms}$, followed by a fixation cross which stayed onscreen for $850 \mathrm{~ms}$. After this period, there was a simultaneous onset of all stimuli. The stimuli were presented for a brief exposure time (47-82 ms), adjusted individually for each observer (see below), and then replaced by a mask (see Fig. 3 for details). The mask at each stimulus location consisted of ten lines of a width of $0.1^{\circ}$ and a randomly chosen length between $0.14-1.7^{\circ}$. They were distributed uniformly, with their center no further than $1.7^{\circ}$ away from the center of the (invisible) array cell. Orientation of each line segment was also determined randomly.

The mask stayed on the screen until observers had reported the location of the target (left or right half of the display). Then the screen went blank until observers rated the confidence in their response. After an erroneous 


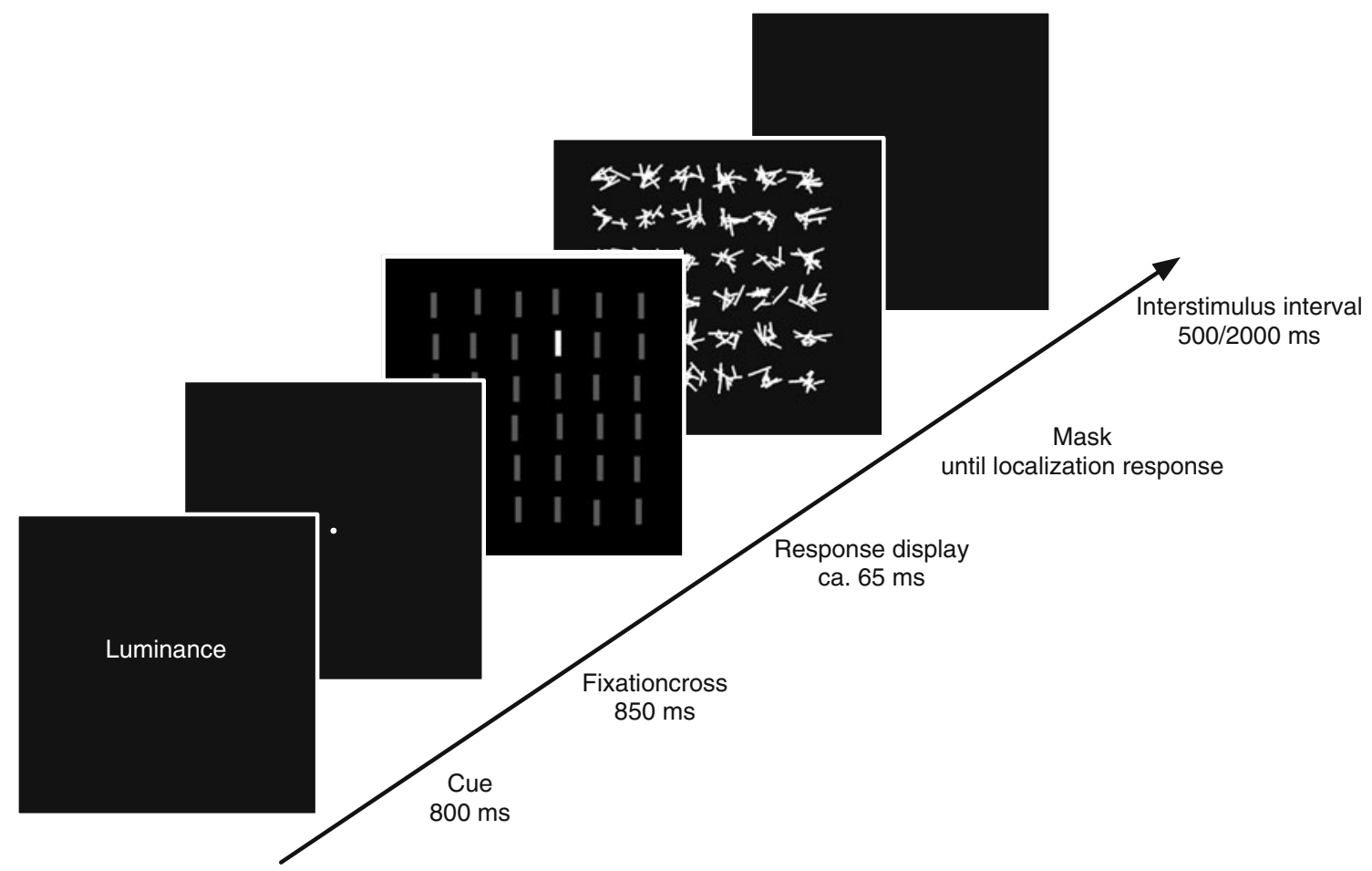

Fig. 3 The sequence of events during one exemplary trial of Experiment 1, in which a luminance feature singleton was validly cued

response, the screen remained blank for a further period of $2 \mathrm{~s}$, providing a feedback signal. On correct trials, the intertrial interval was $0.5 \mathrm{~s}$.

Design and procedure The experiment consisted of two sessions. Before the experimental trials in the first sessions, for each observer, presentation time and intensity of luminance targets were determined by an adaptive staircase procedure (without cue presentation) - such that, on average, performance was correct in $80 \%$ of the trials (Johnston, Cumming, \& Parker, 1993). At this level (in between guessing and ceiling level accuracy), dimensional weighting effects were considered to have a reasonable chance to modulate performance. The presentation time and luminance (intensity) parameters were determined in two phases. In the first, only orientation targets were presented and the presentation duration was estimated that would yield a response accuracy of $80 \%$. In the second phase, the duration determined in the first phase was used to adapt the level of intensity required for luminance-defined targets to reach the $80 \%$ criterion.

These settings were then introduced in the subsequent experimental trials in which observers performed both the neutral and the valid/invalid-cue conditions. In these trials, a cue was always presented, and observers were informed that orientation/luminance cues correctly indicated the dimension of the upcoming target with a probability of $80 \%$ (while neutral cues predicted it with a probability of
$50 \%$ ). Sessions consisted of sequences of five blocks, one with neutral and four with valid/invalid dimension cues. Individual observers started at a random point in this sequence. Blocks consisted of 60 trials, followed by performance feedback. The sessions lasted $50 \mathrm{~min}$ each, and they were separated by at least $2 \mathrm{~h}$ but not more than 2 days.

\section{Results}

Data analysis was performed using R (R Development Core Team, 2007). For the analysis of accuracy dependent on the target-defining dimension on the preceding trial, trials were excluded if there had been an incorrect response on trial $n-1$, about $21 \%$ of trials overall. The high error rate showed that the manipulation of presentation time was successful and adequate for a signal detection task. As accuracy was the dependent variable, it was unnecessary to remove error trials (n) from the analysis. However, for the examination of dimensional intertrial transitions, it was necessary to remove trials ( $n$ ) that were preceded by an error (on trial $n-1$ ), so as to make sure that participants had processed the target on trial $n-1$ correctly. Figure 4 presents percent correct (pc) for the different types of cue and intertrial transition conditions, respectively.

Mean percent correct (pc) was $78.3 \%$. Pc varied with confidence: accuracy was 59,76 , and $87 \%$ for decisions of 
Fig. 4 Accuracy for invalid, neutral, and valid cues (left panel) and for dimension switches (different dimension $d D$, same dimension $s D$; right panel)

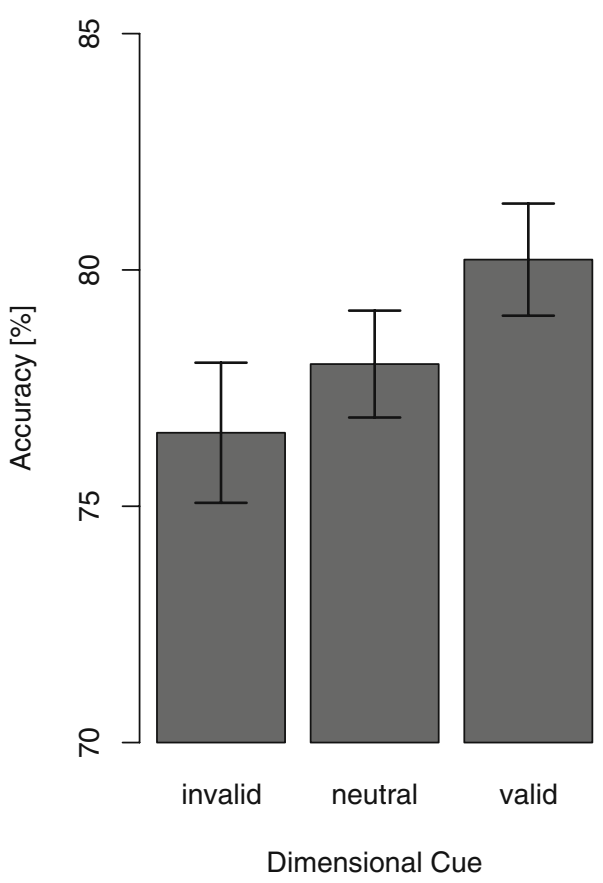

low, medium, and high confidence, respectively. A repeated-measures analysis of variance (ANOVA) of the pc scores, with the factors target dimension (orientation, luminance), cue validity (neutral, valid, invalid), and intertrial transition (same, different dimension), revealed the main effects of cue validity $[F(2,50)=10.7, p<.0001]$ and intertrial transition $[F(1,25)=14.3, p<.001]$ to be significant (no further effects were significant). Response accuracy was lower on invalid- compared to neutral- and valid-cue trials (76.5 vs 78.0 and $80.2 \%$; main effect of cue validity). A priori planned one-sided $t$ tests (Bonferronicorrected) revealed accuracy to be higher for valid cues than for both neutral and invalid cues (both $p<.001$ ), whereas the difference between neutral and invalid cues was statistically not reliable $(p>.15)$. Furthermore, accuracy was higher for trials that were preceded by a target defined in the same, rather than a different, dimension (79.4 vs 77.0\%; main effect of intertrial transition).

\section{Discussion}

In contrast to predictions of dual-route or post-selective accounts, Experiment 1 demonstrated significant dimensional intertrial (repetition/change) effects (i.e., a cost of about $3 \%$ for dimension change vs repetition trials) as well as dimensional cueing effects (i.e., a benefit of about $4 \%$ for valid- relative to invalid-cue trials). Note that feature-specific intertrial effects, caused by a change of the target-defining feature within a repeated dimension, are generally smaller than dimension-specific effects and typically non-significant for orientation targets if the distractor features remain constant (e.g., Found \& Müller, 1996; Huang \& Pashler, 2005; Krummenacher, Grubert, \& Müller, 2010; Müller et al., 2003). For this reason, it has become a common practice to use only one feature per dimension (see, e.g., Töllner et al., 2008, or Mortier et al., 2010). Although in Experiment 1 there were two features for the orientation dimension, the precise target orientation was not recorded, so that it was not possible to compare the size of dimension change to that of feature change effects.

The localization task used would have made the dimensional modules assumed in the dual-route model (see Fig. 2) useless, as these are, by definition, non-spatial detection mechanisms; and it would have rendered the post-selective weighting mechanisms proposed by the DAM (Cohen \& Magen, 1999) ineffective because the search- (feature contrast in the orientation or in the luminance dimension) and response-defining (left or right half of the display) features were dissociated (cf. Bravo \& Nakayama, 1992; Duncan, 1985). Finally, the brief presentation time and use of an accuracy measure ensured that the dimensional effects originated from perceptual processing stages, rather than stages related to response selection or response execution (Santee \& Egeth, 1982; Huang \& Pashler, 2005; Ester \& Awh, 2008; Sigurdardottir et al., 2008).

This set of findings is in line with the one-route account of pre-selective dimensional weighting, according to which dimensional weights can be allocated passively, in bottom-up fashion (Found \& Müller, 1996; Müller et al., 1995) as well as actively by top-down intention (Müller \& Krummenacher, 2006; Müller et al., 2003), whether the task requires simple detection or localization of the feature singleton. 
There is, however, one difference to previous reports of dimensional cueing effects: Müller et al. (2003) found an interaction between cue type and dimension sequence such that the intertrial effects were larger for neutral-cue trials than for valid- and invalid-cue trials (without a difference between the latter), indicative of a top-down modulation of dimension switch costs (e.g., setting oneself in advance to the cued dimension on a given trial reduces the cost associated with a dimension change relative to the preceding trial). Probably, the generally small numerical size of the dimensional intertrial effects in Experiment 1 made such a modulation hard to observe.

\section{Experiment 2}

Experiment 2 was designed to examine whether a second manipulation of salience, namely, reduction of feature contrast (as compared to the limited presentation times in Experiment 1), would render dimensional sequence and cueing effects observable in a speeded localization task under unlimited viewing conditions.

In a field of homogeneous distractors, increasing the similarity of the target to the distractors is known to reduce search performance (Duncan \& Humphreys, 1989; Nothdurft, 1993; Sato, Murthy, Thompson, \& Schall, 2001; Schall \& Thompson, 1999). Thus, for the reasons outlined above, the target feature contrast was reduced in one (low-contrast) condition of Experiment 2 by increasing target-distractor similarity in terms of orientation and luminance, compared to a high-contrast condition. Importantly, this reduction was such that it preserved search efficiency measured in terms of the slope of the function relating detection RTs to set size ( $\leq 2 \mathrm{~ms} /$ item, indicative of 'efficient'; see Appendix for details).

Additionally, we observed that many participants actually failed to remember the instruction to make use of the cue, and attributed little use to doing so. Therefore, it is quite possible that participants simply failed to make use of the cues in some studies (in particular, when performance was not limited by saliency-based selection). To prevent this, Müller \& Krummenacher (2006) asked their observers to rate, after some randomly selected trials, how well they had made use of the cue, thereby making it unlikely that they forgot this part of the instruction. This procedure has been criticized as introducing a dual-task situation where observers additionally had to monitor and remember how they use the cues on a trial. To avoid this, we used a standardized protocol for instructing the usage of dimensional cues which included double-checking how the instruction was actually understood by observers and repeating the instruction if necessary (see details below).
Method

Participants A total of 12 observers participated in Experiment 2 (4 male; median age 23 years; all right-handed; and all with normal or corrected to normal vision). Observers were paid at a rate of Euro 8.00 per hour for their service.

Apparatus, stimuli, and timing The apparatus was the same as in Experiment 1. Distractors were 34 vertical green bars. The bars were arranged on three (invisible) circles with a radius of $4.5,8.5$, and, respectively, $12.5^{\circ}$ of visual angle around a $0.2^{\circ}$ white fixation spot. The inner circle consisted of 6 , the middle circle of 12 , and the outer circle of 16 items. Targets could be placed on positions 2, 3, 4 (right half of the display) and positions 8, 9, or 10 (left half) on an imaginary clock superimposed on the middle circle. The bars extended $0.3^{\circ}$ in width and $1.3^{\circ}$ in height. A target was present on each trial. Targets were either orientationdefined, tilted either $45^{\circ}$ (high contrast) or $10^{\circ}$ (low contrast) randomly to the left or right from the vertical; or targets were color-defined, differing from distractors (green; CIE coordinates $0.289,0.494$; luminance $7.3 \mathrm{~cd} / \mathrm{m}^{2}$ ) by either a low color contrast (yellowish green; CIE coordinates $0.344,0.437$; luminance $7.1 \mathrm{~cd} / \mathrm{m}^{2}$ ) or a high contrast (red targets; CIE coordinates $0.462,0.370$; luminance $7.1 \mathrm{~cd} / \mathrm{m}^{2}$ ). These orientation and color target features were chosen based on a pilot experiment, in order to ensure that there was no RT difference between dimensions, but only one between the different levels of feature contrast within each dimension. A set size experiment (see Appendix) confirmed search to be efficient even for low feature contrast targets (search slope less than $2 \mathrm{~ms} /$ item).

Design and procedure In Experiment 2, the target-defining dimension (orientation or color), feature contrast of the target (low or high), and validity of dimensional pre-cues (valid or neutral) were manipulated. The target was equally likely to be orientation- or luminance-defined, and to be of low or high feature contrast. Of 52 trials in each block, 10 presented neutral and 42 valid cues (i.e., there were $20 \%$ neutral and $80 \%$ valid cues per block; a dimension cue, i.e., the cue 'orientation' or 'color', was never invalid). In order to provide ideal conditions for observers to utilize the cue and actively set themselves to the indicated dimension, the length of the experiment was limited to six blocks (i.e., 312 ) trials (as use of the cue is not necessary to perform the task and not gainful subjectively, cue utilization would be expected to decrease with practice of the task). As a result of this, the number of observations per condition ranged from just 10 to 15 for the neutral cue conditions (after removal of errors). Due to the low number of observations in some conditions, RTs were not filtered for outliers and medians were used as the best estimator of each observer's 
RT performance in each condition. For the analysis of the cue validity effect, trials were pooled across dimension repetition and change conditions. And for analyzing the dimension sequence effect, trials were pooled across the three cue validity conditions. The low number of trials made an analysis of the interaction between cue and dimension sequence impossible.

Observers received written instructions about the presentation and validity of the cues, and they were asked (1) to indicate the location of the feature singleton as rapidly as possible and (2) actively prepare themselves for a singleton in the pre-cued dimension even though dimension was irrelevant for the required (localization) response. Each observer was asked, in a standardized fashion, to repeat the instruction verbally after having read it. Following this, it was emphasized to participants that they were to make consistent use of the cues even though the task could be solved without taking the cue into account (observers' responses to this second instruction were noted as well). While all participants correctly reproduced the written instruction that their task was to localize the target, none of them reproduced the instruction to make use of the cues. After verbal re-instruction, six participants related voluntarily that they thought the cue to be of no help; to these observers, the experimenter repeated for a second time that they should make use of the cue even though it was not response-relevant.

\section{Results and discussion}

Error rates and RTs. Overall error rates were low (2.2\%). An ANOVA of the error rates with the factors dimension (color, orientation) and contrast (low, high) revealed that observers performed better when feature contrast was high compared to low: 1.0 versus $3.3 \%$ (main effect of feature contrast: $F(1,11)=0.9, p<.001)$. There was also a trend for contrast affecting performance more with orientation ( 1 vs $4 \%$ ) than with color targets ( 1 vs $2.5 \%)[F(1,11)=4.5$, $p=.06]$. Error rates were not statistically different for valid and neutral cues $(2.0$ vs $2.1 \% ; p>9)$ and for dimension repetitions and changes $(2.3$ vs $2.1 \% ; p>.73)$.

The effects of dimensional pre-cue and dimension repetition/change were analyzed in two different ANOVAs, as there were not enough trials for the full factorial analysis. The first ANOVA of the RTs, with the factors dimension, feature contrast, and cue, revealed significant main effects of feature contrast $[F(1,11)=113.9, p<.0001]$ and cue $[F(1,11)=10.9, p<.01]$. High-contrast targets were responded to faster than low-contrast targets (360 vs $428 \mathrm{~ms}$ ), independently of the target-defining dimension. Furthermore, RTs were faster for valid- than for neutral-cue trials (386 vs $402 \mathrm{~ms}$ ). Most importantly, the two-way interaction of feature contrast with cue validity was significant $[F(1,11)=5.0, p<.05]$. For high-contrast targets, cueing benefits were $10 \mathrm{~ms}$ (not significantly different from zero: $t(11)=1.8, p<.1$ ) ; in contrast, for low-contrast targets, the cueing benefits were $23 \mathrm{~ms}$ [significantly greater than zero: $t(11)=3.9, p<.01$ ]

The second ANOVA, with the factors dimension, feature contrast, and dimension repetition/change also revealed a significant main effect of contrast $[F(1,11)=100, p<.0001]$ as well as of dimension change $[F(1,11)=11.7, p<.01]$; additionally, the interaction of dimension change with contrast was significant $[F(1,11)=11.1, p<.01]$. Dimension repetitions led to faster RTs than changes ( 383 vs $393 \mathrm{~ms}$ ). Dimension repetition/change effects were not significant for high-contrast targets [1 ms: $t(11)=0.6, p>.6]$, but significant for low-contrast targets [18 ms, $t(11)=.38, p<.01]$.

Experiment 2 was motivated by the question whether dimensional cueing effects and intertrial repetition/change effects would re-emerge in a speeded localization task when the saliency of feature singleton targets is well below maximum. Target saliency was manipulated by varying the similarity of target to the distractor features. The results revealed a difference in latencies between the two levels of feature contrast of the order of $70 \mathrm{~ms}$, without making search inefficient (search slopes $\leq 2 \mathrm{~ms} /$ item; see Appendix). In contrast to predictions of dual-route models (the task required spatial information) and of post-selective accounts such as the DAM (the target-defining and response-defining features were independent), there were significant dimension repetition/change and dimensional cueing effects. These findings are in line with the pre-selective one-route model of dimension weighting if the non-linear, asymptotic behavior of salience (Gao et al., 2008; Nothdurft, 1993) is taken into account.

\section{General discussion}

\section{Summary of findings}

The present study examined two alternatives to the preattentive, one-route DWA: dual-route (e.g., FIT) and postselective weighting models (e.g., DAM). The task employed required coarse localization of a feature singleton (left vs right hemi-field) under two conditions restricting the perceptual quality of the target (brief presentation times in Experiment 1 and reduced feature contrast in Experiment 2). Under both conditions, significant dimension intertrial and cueing effects were observed. Dual-route models cannot account for this finding, because a localization task necessarily involves spatial information which, according to Chan and Hayward (2009; see also Mortier et al., 2010), is processed via an unweighted saliency map, rather than weighted non-spatial 
dimensional modules (Fig. 2). And dimensional weighting effects were not expected on the DAM because the targetand response-defining features are uncorrelated in a localization task.

Accuracy modulations due to dimensional weighting under brief viewing conditions generally suggest perceptual, rather than post-selective, weighting mechanisms to be in operation, because under brief viewing conditions post-selective processing stages have been 'saturated' (Santee \& Egeth, 1982). Perceptual processing stages, however, involve post-selective processing (such as focalattentional stimulus identification) as well as pre-attentive processing, and the DWA attributes dimensional cueing and repetition/change effects to pre-selective perceptual processing stages.

\section{Relations to other studies}

The present findings can be related to two classes of paradigms which have been used to investigate sequence effects and the role of intention in visual search: the dimension-change and the target/distractor (TD) featureswap paradigm. In the dimension-change paradigm, as in the present study, the distractor items are constant and the target differs from distractors in one of several possible dimensions (e.g., Found \& Müller, 1996; Theeuwes et al., 2006), whereas in the TD feature-swap paradigm, only one target defining dimension is relevant (e.g., color) and the target and distractors can change roles from trial to trial (such as a red among green or a green amongst red items; e.g., Maljkovic \& Nakayama, 1994).

Dimension-change paradigm Using a dimension-change paradigm, several studies failed to find significant dimension sequence and/or cueing effects in localization or compound-search tasks, whereas others (including the present study) did observe such effects (see Table 1). What are possible reasons for these discrepant findings? Certainly, the present findings indicate that dimension sequence and cueing effects are more readily observed if stimulus quality is reduced (while search is still efficient), and this is so in the localization task used in Experiments 1 and 2 as well as the simple detection task (see re-analysis of the data of Zehetleitner et al., 2009). This pattern of effects is as expected given the asymptotic growth of salience activations (Nothdurft, 1993, 2000; Gao et al., 2008).

Although the present findings do not account for the exact differences in processing between non-spatial detection and spatial compound and localization tasks, they limit the feasible alternatives. One view holds that spatial and non-spatial 'pop-out' tasks are based on fundamentally different processing architectures, which would rule out the existence of dimensional effects in spatial tasks in principle
(Chan \& Hayward, 2009; Mortier et al., 2010). One other prediction deriving from this view is that interference from cross-dimensional additional singletons, which are known to impair search performance in compound tasks (e.g., Theeuwes, 1992), cannot in principle occur in detection tasks, because the latter are not processed via the salience map route. Consistent with this idea, there have been several null-findings of interference from cross-dimensional additional singletons in detection tasks (Kumada, 1999; Chan \& Hayward, 2009). However, by using less salient targets and by reducing the frequency of occurrence of additional singletons, interference effects do become demonstrable in detection tasks. Importantly, Zehetleitner, Proulx, and Müller found the magnitude of interference to be dependent on the spatial distance between target and additional singleton (in the same way as in compound tasks)_-providing further support for a salience map-based processing architecture for detection tasks.

If the difference in processing between detection and compound/localization tasks is not one of qualitatively different processing architectures, it may instead be one of a matter of degree. Consistent with this is the fact that manipulations of salience, rather than simply leading to either the presence or the absence of dimension-based effects (as in the present study), give rise to a graded modulation of dimensional effects. Thus, in the detection task of Zehetleitner et al., (2009), for example, dimension change effects were reduced for targets of high salience, rather than being entirely abolished. In addition, a different type of salience-based effect, gains due to redundant target definition in two dimensions, was also modulated by feature contrast; the gains were smaller, though not abolished, with high as compared to low levels of feature contrast. Taken together, these findings suggest that not only the size of dimensional intertrial effects but also that of other salience-based effects varies in a graded fashion as a function of feature contrast.

But even so, this does not explain why dimension-based effects are measurable with standard, high-salience targets in detection tasks but not in localization tasks. It is quite unlikely that stimulus salience itself is affected by the task. However, conceivably, the speed of perceptual decisions differs between both tasks. For example, in signal detection terms, there is more information in one and the same display for a two-alternative forced-choice (2AFC) localization decision than for a yes/no detection decision. The reason is that, in the localization task, either the left or the right half of the display contains positive evidence of target presence and, simultaneously, the respective other half yields negative evidence for target absence (see Macmillan \& Creelman, 2005). According to Macmillan \& Creelman (2005); see equations 7.5 and 7.6 on p. 171), the maximum proportion of correct responses, $p(c)_{\max }$, is $p(c)_{\max \text { yes-no }}=$ 
$\Phi\left(d^{\prime} / 2\right)$ in the yes/no detection task, whereas it is $p(c)_{\max 2 A F C}=\Phi\left(d^{\prime} / \sqrt{2}\right)$ in the 2AFC localization task. Accordingly, it can be calculated that, with a value of, say, $\mathrm{d}^{\prime}=1$, proportion correct is about $70 \%$ in a yes/no detection task, but $76 \%$ in a $2 \mathrm{AFC}$ localization task. With speeded RT tasks, the increase in information for localization tasks would lead to faster decisions compared to detection tasks. It is known that the same difference in stimulus quality yields larger differences in decision times (and, thus, in RT) for fast than for slow decisions (Thapar, Ratcliff, \& McKoon, 2003; see their Fig. 1), and decision times are slower for lowsalience targets than for high-salience targets. ${ }^{1}$ Although thus far only speculative, the idea that decision speed can modulate salience-based effects is supported by Experiment 2 of Zehetleitner et al. (2009): manipulating speed-accuracy trade-off, they found that participants who had shorter decision times (and made more mistakes) exhibited smaller redundancy gains than those with longer decision times (and fewer mistakes).

Relatedly, Mortier et al. (2010) reported equivalent RTs for detection or localization of feature singletons. However, it is not possible to infer from equal RTs that the speed of the perceptual decision is equal; this is because RTs are composites of decision times and processing times for response selection and execution. In fact, Zehetleitner and Müller (2010) have recently observed that, for efficient search, the RT difference caused by two levels of salience or by redundancy of the target definition were approximately twice as large for detection as for localization tasks, while simultaneously localization RTs were about $80 \mathrm{~ms}$ faster than detection RTs.

Taken together, it is conceivable that localization decisions are faster than detection decisions, which is why modulations of salience by dimension sequence or dimensional pre-cueing are smaller (and sometimes not even measurable) for localization than for detection tasks. On this view, the factor that is the cause of the differences in observed salience effects (whether redundant target definition, dimension sequence, or dimension cueing) between detection and localization tasks is decision speed, rather than the task itself. This proposal is theoretically parsimonious, as it can explain modulations of effects both within tasks (as in Zehetleitner et al., 2009) and across tasks (detection and localization). Note, though, that this explanation is, in the first instance, limited to 2-alternative forced-choice detection and localization tasks. In a compound task, the target has to be localized at one out of $n$

\footnotetext{
${ }^{1}$ In diffusion models (e.g., Thapar et al., 2003), RTs are considered to be the sum of two random variables, a decisional component corresponding to the perceptual decision of SDT paradigms and a non-decision component related to response selection and execution. The diffusion process, that is, the accumulation of evidence, models the speed of the perceptual decision.
}

possible positions (for display size of $n$ ), and it remains unclear how $n$-alternative localization decisions can be formed faster than 2-alternative yes/no detection decisions.

\section{TD feature-swap paradigm}

Finally, it is interesting to consider how the present findings of dimension-based effects relate to the effects demonstrated using a TD feature-swap paradigm. Featural effects in this paradigm have several distinct characteristics: (1) they generally occur in compound-search tasks (except in a few studies, e.g., Kristjansson, Wang, \& Nakayama, 2002, who used a conjunction search task; Kristjánsson, 2006, Experiment 4; and Wolfe, Butcher, Lee, \& Hyle, 2003); (2) they enter into strong interactions with the response-defining feature (Becker, 2008b; Hillstrom, 2000; Huang, Holcombe, \& Pashler, 2004); (3) they are thought to modulate the speed of allocating focal attention to the target (Goolsby \& Suzuki, 2001), as also evidenced by modulations of the latency of the N2pc component of the event-related potential (Eimer, Kiss, \& Cheung, 2009); (4) they can be modulated by cueing (Folk \& Remington, 2008; Leonard \& Egeth, 2008); and (5) they are thought to vary with target 'ambiguity' or salience (Meeter \& Olivers, 2006; Olivers \& Meeter, 2006). Taken together, all these six aspects also hold for the dimensionchange paradigm: the effects can be found in non-detection (e.g., compound or localization) tasks (Töllner et al., 2008; present study); they can interact with the response-defining feature (e.g., Chan \& Hayward, 2009; Müller \& Krummenacher, 2006); they are thought to modulate the speed of selection (dimension-weighting account), as reflected in modulations of the N2pc latency (Töllner et al, 2008, 2010); they can be modulated by cueing (Müller et al., 2003); and they vary with salience (present study).

Yet, despite these similarities, it remains that effects which are reliably reported for the TD feature-swap paradigm sometimes fail to be observed in the dimensionchange paradigm (e.g., Chan \& Hayward, 2009; Mortier et al., 2010; Theeuwes et al., 2006). However, given the similarities, again, the difference between the two types of paradigm is likely to be a matter of degree, rather than a fundamental one - though the exact nature of the difference needs to be investigated in future studies.

\section{Conclusion}

In summary, the present findings demonstrate the existence of dimensional sequence and pre-cueing effects in a localization task under conditions of reduced target saliency, which (due to the asymptotic function of the saliency activation) are more conducive to such effects becoming observable. Salience was reduced by limited presentation time 
in Experiment 1 and by decreasing target feature contrast in Experiment 2 . The present findings do not conclusively answer why the same (high) level of salience gives rise to reliable dimensional effects in detection tasks, but less so in compound and localization tasks. However, they limit possible explanations, in particular: qualitative differences in the processing architecture, as assumed by DAM (e.g., Cohen \& Shoup, 2000) or a strong FIT (e.g., Chan \& Hayward, 2009), cannot be responsible for reported failures to find dimensional effects in compound/localization tasks-because if there were such qualitative differences, no dimensional effects should have been observed in the present localization task with reduced target salience. As an alternative, we tentatively propose that longer decision times lead to larger salience-based effects (including redundancy gains and dimensional effects: Zehetleitner et al., 2009; see also Thapar et al., 2003). This would mean, however, that localization decisions are reached faster than detection decisions.

Author Note This research was supported by grants from DFG Excellence Cluster EC 142 'CoTeSys' (H. J. Müller \& M. Zehetleitner), the DFG research group FOR480 (H. J. Müller), and the Swiss National Science Foundation (PP001 - 110543/1, J. Krummenacher). Correspondence concerning this article should be addressed to Michael Zehetleitner.

\section{Appendix}

Experiment A1 introduced a set size manipulation to examine whether or not the low feature contrast targets used in Experiment 2 are found 'efficiently'. The benchmark was Wolfe's (1998) criterion for 'efficient' search, namely: a search rate less than $5 \mathrm{~ms} /$ item (for rates between 5 and $10 \mathrm{~ms} /$ item, Wolfe considers search to be 'rather efficient').

\section{Experiment A1}

\section{Method}

Participants Eight observers participated in Experiment A1 (5 male; median age 26.5 years; all right-handed; and all with normal or corrected-to-normal vision).

Apparatus, stimuli, and timing The apparatus was the same as in Experiment 2, and stimulus properties (size, color, and orientation) were the same as for the low-feature-contrast targets in Experiment 2. Stimuli were arranged on three (invisible) circles with the same radii and number of stimulus positions as in Experiment 2. In contrast to Experiment 2, there was one further stimulus position in the center of the screen, and a target could also appear on the inner circle and the center position (i.e., 19 possible positions) (rather than only on the positions of the middle circle, as was the case in Experiment 2). Targets could differ from distractors in terms of color and orientations, with low levels of feature contrast (for details, see Experiment 2).

The main manipulation in Experiment A1 was set size: large or small. In the small-set-size condition, displays did not contain the outer ring of stimuli; to ensure that targets were surrounded by six distractors (as was the case in the large set condition and Experiment 2), targets could then be placed on one of the positions on the inner circle or the center position only (i.e., 7 possible target positions). In order to keep the density of the stimuli constant across the two set size conditions, while at the same time keeping the positional probability of target occurrence constant, the small-set-size display could be presented centered at any of six equidistant positions $4.5^{\circ}$ from the middle of the screen.

Design and procedure In Experiment A1, target dimension (orientation vsp. color) and set size (19 vsp. 7) were manipulated. The target was equally likely to be orientation- or color-defined. A target was present in 50\% of all trials. Trials were presented in 3 blocks of 80 trials each. Observers received written instructions to press the right button of a mouse if a target was present (irrespective of set size or dimension) and to withhold a response if no target was present (go/no-go task). In case of target absence, the next trial was initiated $900 \mathrm{~ms}$ after stimulus onset, provided that no response was registered.

\section{Results and discussion}

There were no trials with (target-present) responses faster than $150 \mathrm{~ms}$, so no anticipatory-response trials had to be removed prior to analysis.

The error rate was low overall (1.5\%), with $2.0 \%$ false alarms and $1.0 \%$ misses. An ANOVA of the error rates with the factors target type (absent, color, or orientation) and set size (small vs. large) revealed no significant effects (all $F<1.7, p>$.2).

An analogous ANOVA of the reaction times on correctresponse trials (factor target type: color or orientation) also revealed no significant effects (all $F<3.0$, all $p>.12$ ). Separate analyses of the search rates (in $\mathrm{ms} /$ item) for colorand orientation-defined targets revealed all rates (1.2 and $1.4 \mathrm{~ms} /$ item, respectively) to be significantly below $5 \mathrm{~ms} /$ item [both $t(7)<-3.8$ and $p<.01)$.

In summary, Experiment A1 verified that even targets of low feature contrast could be searched for efficiently. 


\section{References}

Becker, S. I. (2008a). Can intertrial effects of features and dimensions be explained by a single theory? Journal of Experimental Psychology: Human Perception and Performance, 34, 14171440. doi:10.1037/a0011386

Becker, S. I. (2008b). The stage of priming: Are intertrial repetition effects attentional or decisional? Vision Research, 48, 664-684. doi:10.1016/j.visres.2007.10.025

Bravo, M. J., \& Nakayama, K. (1992). The role of attention in different visual-search tasks. Perception \& Psychophysics, 51, 465-472.

Bruce, N. D. B., \& Tsotsos, J. K. (2009). Saliency, attention, and visual search: An information theoretic approach. Journal of Vision, 9(5), 1-24. doi:10.1167/9.3.5

Chan, L. K. H., \& Hayward, W. G. (2009). Feature integration theory revisited: Dissociating feature detection and attentional guidance in visual search. Journal of Experimental Psychology: Human Perception and Performance, 35, 119-132. doi:10.1037/00961523.35.1.119

Cohen, A., \& Feintuch, U. (2002). The dimensional-action system: A distinct visual system. In Common mechanisms in perception and action: Attention and performance XIX (pp. 587-608). New York: Oxford University Press.

Cohen, A., \& Magen, H. (1999). Intra-and cross-dimensional visual search for single-feature targets. Perception \& Psychophysics, 61, 291-307.

Cohen, A., \& Shoup, R. (1997). Perceptual dimensional constraints in response selection processes. Cognitive Psychology, 32, 128-181.

Cohen, A., \& Shoup, R. (2000). Response selection processes for conjunctive targets. Journal of Experimental Psychology: Human Perception and Performance, 26, 391-411.

Duncan, J. (1985). Visual search and visual attention. In M. I. Posner \& O. Marin (Eds.), Attention and Performance XI (pp. 85-106). Hillsdale: Lawrence Erlbaum.

Duncan, J., \& Humphreys, G. (1989). Visual search and stimulus similarity. Psychological Review, 96, 433-458.

Eimer, M., Kiss, M., \& Cheung, T. (2009). Priming of pop-out modulates attentional target selection in visual search: Behavioural and electrophysiological evidence. Vision Research, in press, doi:10.1016/j.visres.2009.11.001

Ester, E., \& Awh, E. (2008). The processing locus of interference from salient singleton distractors. Visual Cognition, 16, 166-181.

Evans, K. K., \& Treisman, A. (2005). Perception of objects in natural scenes: Is it really attention free? Journal of Experimental Psychology: Human Perception and Performance, 31, 14761492. doi:10.1037/0096-1523.31.6.1476

Folk, C. L., \& Remington, R. W. (2008). Bottom-up priming of topdown attentional control settings. Visual Cognition, 16, 215-231. doi:10.1080/13506280701458804

Found, A., \& Müller, H. J. (1996). Searching for unknown feature targets on more than one dimension: Investigating a "dimensionweighting" account. Perception \& Psychophysics, 58, 88-101.

Gao, D., Mahadevan, V., \& Vasconcelos, N. (2008). On the plausibility of the discriminant center-surround hypothesis for visual saliency. Journal of Vision, 8, 1-18.

Goolsby, B. A., \& Suzuki, S. (2001). Understanding priming of colorsingleton search: Roles of attention at encoding and "retrieval". Perception \& Psychophysics, 63, 929-944.

Hillstrom, A. P. (2000). Repetition effects in visual search. Perception \& Psychophysics, 62, 800-817.

Huang, L., Holcombe, A. O., \& Pashler, H. (2004). Repetition priming in visual search: Episodic retrieval, not feature priming. Memory \& Cognition, 32, 12-20.
Huang, L., \& Pashler, H. (2005). Expectation and repetition effects in searching for featural singletons in very brief displays. Perception \& Psychophysics, 67, 150-157.

Itti, L., \& Koch, C. (2001). Computational modelling of visual attention. Nature Reviews. Neuroscience, 2, 194-203.

Johnston, E., Cumming, B., \& Parker, A. (1993). Integration of depth modules: Stereopsis and texture. Vision Research, 33, 813-826.

Koch, C., \& Ullman, S. (1985). Shifts in selective visual attention: Towards the underlying neural circuitry. Human Neurobiology, 4, 219-227.

Kristjánsson, A. (2006). Simultaneous priming along multiple feature dimensions in a visual search task. Vision Research, 46, 2554 2570. doi:10.1016/j.visres.2006.01.015

Kristjansson, A., Wang, D., \& Nakayama, K. (2002). The role of priming in conjunctive visual search. Cognition, 85, 37-52.

Krummenacher, J., Grubert, A., \& Müller, H. J. (2010). Inter-trial and redundant-signals effects in visual search and discrimination tasks: Separable pre-attentive and post-selective effects. Vision Research, 50, 1382-1395.

Kumada, T. (1999). Limitations in attending to a feature value for overriding stimulus-driven interference. Perception \& Psychophysics, 61, 61-79.

Kumada, T. (2001). Feature-based control of attention: Evidence for two forms of dimension weighting. Perception \& Psychophysics, 63, 698-708.

Leonard, C. J., \& Egeth, H. E. (2008). Attentional guidance in singleton search: An examination of top-down, bottom-up, and intertrial factors. Visual Cognition, 16, 1078-1091.

Macmillan, N., \& Creelman, C. D. (2005). Detection Theory - A User's Guide. New Jersey: Lawrence Erlbaum.

Maljkovic, V., \& Nakayama, K. (1994). Priming of pop-out: I. Role of features. Memory \& Cognition, 22, 657-672.

Meeter, M., \& Olivers, C. (2006). Intertrial priming stemming from ambiguity: A new account of priming in visual search. Visual Cognition, 13, 202-222. doi:10.1080/13506280500277488

Mortier, K., Theeuwes, J., \& Starreveld, P. (2005). Response selection modulates visual search within and across dimensions. Journal of Experimental Psychology: Human Perception and Performance, 31, 542-557. doi:10.1037/0096-1523.31.3.542

Mortier, K., Zoest, W. V., Meeter, M., \& Theeuwes, J. (2010). Word cues affect detection but not localization responses. Attention, Perception, \& Psychophysics, 72, 65-75. doi:10.3758/APP.72.1.65

Müller, H. J., Heller, D., \& Ziegler, J. (1995). Visual search for singleton feature targets within and across feature dimensions. Perception \& Psychophysics, 57, 1-17.

Müller, H. J., \& Krummenacher, J. (2006). Locus of dimension weighting: Preattentive or postselective? Visual Cognition, 14, 490-513. doi:10.1080/13506280500194154

Müller, H., Krummenacher, J., \& Heller, D. (2004). Dimensionspecific inter-trial facilitation in visual search for pop-out targets: Evidence for a top-down modulable visual short-term memory effect. Visual Cognition, 11, 577-602.

Müller, H. J., Reimann, B., \& Krummenacher, J. (2003). Visual search for singleton feature targets across dimensions: Stimulus- and expectancy-driven effects in dimensional weighting. Journal of Experimental Psychology: Human Perception and Performance, 29, 1021-1035.

Nothdurft, H. C. (1993). The conspicuousness of orientation and motion contrast. Spatial Vision, 7, 341-363.

Nothdurft, H. C. (2000). Salience from feature contrast: Temporal properties of saliency mechanisms. Vision Research, 40, 2421-2435.

Olivers, C., \& Meeter, M. (2006). On the dissociation between compound and present/absent tasks in visual search: Intertrial priming is ambiguity driven. Visual Cognition, 13, 1-28. doi:10.1080/13506280500308101 
Prinzmetal, W., McCool, C., \& Park, S. (2005). Attention: Reaction time and accuracy reveal different mechanisms. Journal of Experimental Psychology: General, 134, 73-92.

R Development Core Team (2007). R: A language and environment for statistical computing. Vienna, Austria: R Foundation for Statistical Computing. ISBN 3-900051-07-0, http://www. R-project.org.

Santee, J., \& Egeth, H. (1982). Do reaction time and accuracy measure the same aspects of letter recognition? Journal of Experimental Psychology: Human Perception and Performance, 8, 489-501.

Sato, T., Murthy, A., Thompson, K., \& Schall, J. (2001). Search efficiency but not response interference affects visual selection in frontal eye field. Neuron, 30, 583-591.

Schall, J., \& Thompson, K. (1999). Neural selection and control of visually guided eye movements. Annual Review of Neuroscience, 22, 241-259. doi:10.1146/annurev.neuro.22.1.241

Sigurdardottir, H. M., Kristjansson, A., \& Driver, J. (2008). Repetition streaks increase perceptual sensitivity in visual search of brief displays. Visual Cognition, 16, 643-658. doi:10.1080/ 13506280701218364

Thapar, A., Ratcliff, R., \& McKoon, G. (2003). A diffusion model analysis of the effects of aging on letter discrimination. Psychology and Aging, 18, 415-429. doi:10.1037/0882-7974.18.3.415

Theeuwes, J. (1992). Perceptual selectivity for color and form. Perception \& Psychophysics, 51, 599-606.

Theeuwes, J., Reimann, B., Mortier, \& Karen. (2006). Visual search for featural singletons: No top-down modulation, only bottom-up priming. Visual Cognition, 14, 466-489. doi:10.1080/ 13506280500195110

Theeuwes, J., \& van der Burg, E. (2007). The role of spatial and nonspatial information in visual selection. Journal of Experimental Psychology: Human Perception and Performance, 33, 13351351. doi:10.1037/0096-1523.33.6.1335
Töllner, T., Gramann, K., Müller, H. J., Kiss, M., \& Eimer, M. (2008). Electrophysiological markers of visual dimension changes and response changes. Journal of Experimental Psychology: Human Perception and Performance, 34, 531-542. doi:10.1037/00961523.34.3.531

Töllner, T., Zehetleitner, M., Gramann, K., \& Müller, H. J. (2010). Top-down weighting of visual dimensions: Behavioral and electrophysiological evidence. Vision Research, 50, 1372-1381. doi:10.1016/j.visres.2009.11.009

Treisman, A., \& Gelade, G. (1980). A feature-integration theory of vision. Cognitive Psychology, 12, 97-136.

Treisman, A., \& Gormican, S. (1988). Feature analysis in early vision: Evidence from search asymmetries. Psychological Review, 95, $15-48$.

Wolfe, J. M. (1994). Guided search 2.0 - a revised model of visual search. Psychonomic Bulletin \& Review, 1, 202-238.

Wolfe, J. M., Butcher, S., Lee, C., \& Hyle, M. (2003). Changing your mind: on the contributions of top-down and bottom-up guidance in visual search for feature singletons. Journal of Experimental Psychology: Human Perception and Performance, 29, 483-502.

Zehetleitner, M., Krummenacher, J., \& Müller, H. J. (2009). The detection of feature singletons defined in two dimensions is based on salience summation, rather than on serial exhaustive or interactive race architectures. Attention, Perception, \& Psychophysics, 71, 1739-1759. doi:10.3758/APP.71.8.1739

Zehetleitner, M., \& Müller, H. J. (2010). Salience from the decision Perspective: you know where it is before you know it is there. Journal of Vision (in press).

Zehetleitner, M., Proulx, M. J., \& Müller, H. J. (2009). Additionalsingleton interference in efficient visual search: A common salience route for detection and compound tasks. Attention. Perception \& Psychophysics, 71, 1760-1770. doi:10.3758/ APP.71.8.1760 